\title{
Emotion Detection on Twitter Data using Knowledge Base Approach
}

\author{
Srinivasu Badugu, PhD \\ Associate Professor \\ Dept. of Computer Science Engineering \\ Stanley College of Engineering and \\ Technology for Women \\ Hyderabad, India
}

\author{
Matla Suhasini \\ Student, M-Tech \\ Dept. of Computer Science Engineering \\ Stanley College of Engineering and \\ Technology for Women \\ Hyderabad, India
}

\begin{abstract}
Emotional states of individuals, also known as moods, are central to the expression of thoughts, ideas and opinions, and in turn impact attitudes and behavior. Social media tools like twitter is increasingly used by individuals to broadcast their day-to-day happenings or to report on an external event of interest, understanding the rich 'landscape' of moods will help us better to interpret millions of individuals. This paper describes a Rule Based approach, which detects the emotion or mood of the tweet and classifies the twitter message under appropriate emotional category. The accuracy with the system is $85 \%$. With the proposed system it is possible to understand the deeper levels of emotions i.e., finer grained instead of sentiment i.e., coarse grained. Sentiment says whether the tweet is positive or negative but the proposed system gives the deeper information of tweet which has adverse uses in the field of Psychology, Intelligence Bureau, Social and Economic trends.
\end{abstract}

\section{General Terms}

Artificial Intelligence, Knowledge Base, Seed List, Twitter Data.

\section{Keywords}

Mood Detection, Emotion, Natural Language Processing, POS Tagging.

\section{INTRODUCTION}

Microblogging is an increasingly popular form of communication on the web. It allows users to broadcast brief text updates to the public or to a selected group of contacts. The development of social network platforms has given people a new way to generate and consume a great deal of information on the web. A large number of social network platforms such as Twitter, Google+, and Facebook provide information for users. Among all Twitter is the most popular microblogging platform in the world. It is also the fastest growing social network platform and has a dominant position in the area of microblogging [1]. Compared with regular microblogging platforms, Twitter messages commonly referred to as tweets are much shorter. This feature makes Twitter easier for people to get the main point from the massive amount of information available online. Twitter users can follow whichever people and information source they prefer [2,3]. Twitter thus has become a powerful platform with many kinds of information from worldwide breaking news to purchasing products at home. Researchers also noticed that tweets often convey pertinent information about the user's emotional states [10]. Emotion analysis on Twitter has thus become an important research issue in the microblogging area
Despite the diversity of uses emerging from such a simple communication channel, it has been noted that tweets normally tend to fall in one of two different content camps: users that microblog about themselves and those that use microblogging primarily to share information [2]. In both cases, tweets can convey information about the mood state of their authors. Even when a user is not specifically microblogging about their personal emotive status, the message can reflect their mood. As such, tweets may be regarded as microscopic instantiations of mood. It follows that the collection of all tweets published over a given time period can unveil changes in the state of public mood at a larger scale.

Emotion/Mood can be expressed in many ways that can be seen such as facial expression and gestures, speech and by written text. The written expression of emotion lacks gestures, tones, and facial expressions, and instead relies on creative use of words for communicating emotion. An increasing number of empirical analyses of sentiment and mood are based on textual collections of data generated on microblogging and social sites. Examples are mood surveys of communication on MySpace, and Twitter. Some of these analyses are focused on specific events, such as the study focused on microbloggers response to the death of Michael Jackson or a political election in Germany, while others analyze broader social and economic trends, such as the relationship between Twitter mood and both stock market fluctuations and consumer confidence and political opinion. The results generated via the analysis of such collective mood aggregators are compelling and indicate that accurate public mood indicators can be extracted from online materials. Using publicly available online data to perform emotion analyses significantly reduces the costs, efforts and time needed to administer large-scale public surveys and questionnaires. These data and results present great opportunities for psychologists and social scientists $[4,5,6]$

\section{RELATED WORK}

There is much less work on emotion analysis in text compared with sentiment analysis.

Some researchers have explored social media such as

Twitter to investigate the potential use of social media to detect depressive disorders. Park [7] ran some studies to capture the depressive mood of users in Twitter. They studied 69 individuals to understand how their depressive states are reflected in their personal updates. The analysis was conducted in three major steps: (1) surveying the users to identify their depression level, (2) collecting tweets of these users, and (3) comparing the depression levels of users with 
their language usage in tweets. They found that social media contains useful signals for characterizing the depression in individuals. Their results showed that participants with depression exhibited increase in the usage of words related to negative emotions and anger in their tweets.

Another work to diagnose depressive disorders in individuals done by Choudhury [8]. They measured behavioral attributes including social engagement, emotion, language and linguistic styles, ego network, and mentions of antidepressant medication. Then they leveraged these behavioral features to build a statistical classifier that estimates the risk of depression. Their models showed an accuracy of $70 \%$ in predicting depression. They have crowdsourced data from Twitter users who have been diagnosed with mental disorders.

Another effort for emotion analysis on Twitter data accomplished by Bollen and his colleagues [2]. They tried to find a relationship between overall public mood and social, economic and other major events. They extracted six dimensions of mood (tension, depression, anger, vigor, fatigue, confusion) using an extended version of POMS (Profile of Mood States), a psychometric instrument. They found that social, political, cultural and economic events have a significant and immediate effect on the various dimensions of public mood.

Recently, Golder studied how individual mood varies from hour-to-hour, day-to-day, and across seasons and cultures by measuring positive and negative effect in Twitter posts, using the lexicon LIWC [9]. Studies were included where a pooled relative measure of an adverse effect (odds ratio or risk ratio) from RCTs could be directly compared, using the ratio of odds ratios, with the pooled estimate for the same adverse effect arising from observational studies. Empirical evidence from this overview indicates that there is no difference on average in the risk estimate of adverse effects of an intervention derived from meta-analyses of RCTs and metaanalyses of observational studies. This suggests that systematic reviews of adverse effects should not be restricted to specific study types.

Some researchers applied lexical approach to identify emotions in text. For example Strapparava and Mihalcea [10] constructed a large lexicon annotated for six basic emotions: anger, disgust, fear, joy, sadness and surprise. In another work, Choudhury [11] identified a lexicon of more than 200 moods frequent on Twitter. Inspired by the circumplex model, they measured the valence and arousal of each mood through mechanical turk and psychology literature sources. Then, they collected posts which have one of the moods in their mood lexicon in the form of a hash-tag at the end of a post.

In [12], three independent annotators manually coded a sample of public tweets and found 9 representative categories including Information Sharing, Self Promotion, Opinions, Random Thoughts, Me Now, Questions to Others, Presence Maintenance, Anecdotes by Me, and Anecdotes by Others. Among them, Me Now (e.g., "tired and upset") and Random Thoughts (e.g., "I miss NY"), frequently displayed profile owner's emotions were the two most popular categories. In order to quantitatively measure depressive symptoms on network level, the authors conducted a longitudinal study on a person-to-person interconnected social network [13] . They assessed over 12,000 people repeatedly over 32 years to retrieve the results. These previous observations give ample ground for using OSN data in studying depressive signs and depression, with the ultimate goal of building a real-time healthcare system.

\section{PROPOSED SYSTEM}

In this paper we have proposed a knowledge Based approach for detecting the emotion or mood of the tweet.

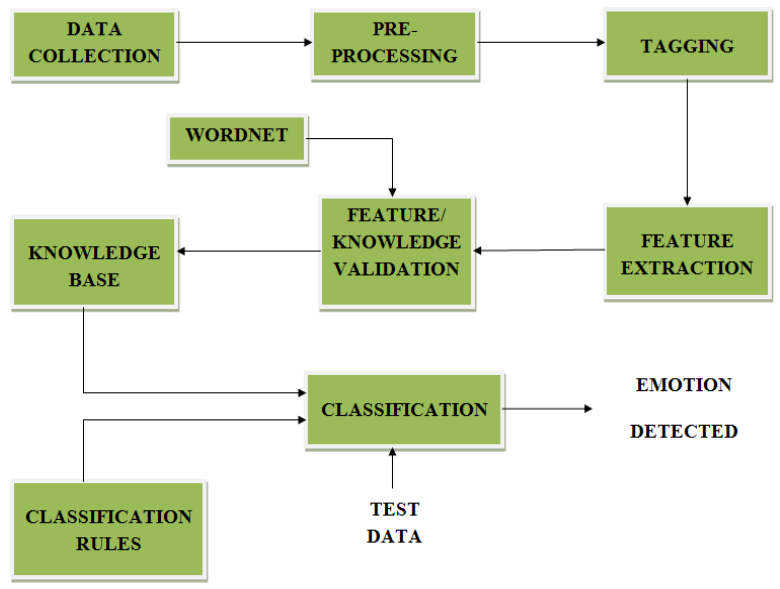

Fig. 1 Architecture of Emotion Detection System

Figure 1 describes the architecture of the system which includes data collection, pre-processing, tagging, knowledge base preparation, knowledge validation and classification.

\subsection{Data Collection}

The data is obtained from Sentiment140 [14], containing 10,48576 tweets. The dataset consists of six attributes the first is polarity, second is id, third is date, fourth is query, fifth is username and sixth is tweet. Considered only the tweet part that is the sixth attribute. In the dataset their purpose is to identify the sentiment, sentiment says whether the tweet is positive or negative but the proposed system purpose is not to identify the sentiment instead it identifies the finer level of emotion.

\subsection{Pre-processing}

Pre-processing is very important to get good results, if there is much noisy, unwanted and unreliable data, then discovering knowledge becomes more difficult. Pre-processing reduces the dimension by removing the unwanted data. This module includes three sub modules such as tokenization, noise elimination and text normalization.

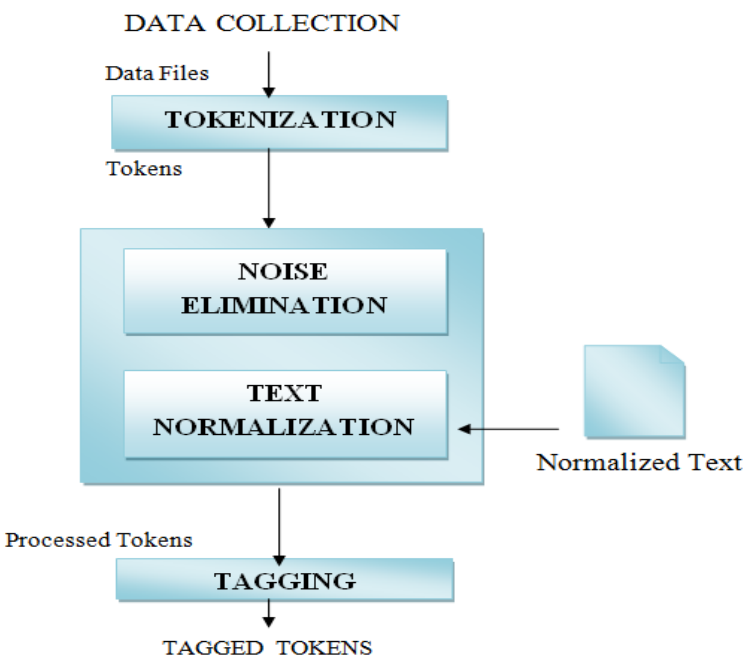

Fig. 2 Workflow of Pre-processing 
3.2.1 Tokenization: Tokenizing using a particular delimiter string, using the split method with space as delimiter the text is made into tokens, as this method is more efficient.

Example:

Input: @ criticalpath Such an awesome idea - the continual learning program with a Kindle http://bit.ly/1ZLfF I am happy 2 hmm sleep.

Output: '@criticalpath', Such', an', awesome',' idea', -',' the', ' continual', 'learning', 'program',' with', 'a', 'Kindle', http://bit.ly/1ZLfF','I','am', 'happy',

2','hmm','sleep'.

3.2.2 Noise Elimination: Analyzing data that has not been carefully screened can produce misleading results. If there is much irrelevant and redundant information or noisy and unreliable data it reduces the performance. Removing such noisy data is preprocessing.

1. Tweets often contain usernames which start with the @ symbol before the username (e.g. @ Marilyn). All such words which starts with @ symbol are removed using regular expression.

2. Many tweets contain URL links. All the URL links are removed using regular expression.

3. Some tweets may contain numeric [0-9], integers are removed using regular expression as the aim is to deal with text data.

4. Tweets may contain special characters such as $(*, /, .,>$ etc ... can be removed.

Strip(): The method strip() returns a copy of the string in which all whitespace characters have to be stripped from the beginning and end of the string (default whitespace characters).

\subsubsection{Text Normalization}

Social media often contains shortcuts, such data is inefficient in further processing and leads to performance degradation, the necessary text should be normalized.

1. The tweets are converted to lower case.

2. The text should be standardized.

Example:

Input: '@criticalpath', Such', an', awesome', idea', -',' the',' continual', 'learning', 'program', with', a', 'Kindle', http://bit.ly/1ZLfF','I','am','happy','

2','hmm','sleep'

Output: 'such', an', awesome',' idea', the', continual',' learning',' program',' with', 'kindle', 'I', 'am',' happy', 'hmm', 'sleep'

\subsection{Tagging}

Tagging is the process of assigning a tag for each token based on the context. Part-of-speech tagging is performed using Python's nltk tagger. POS tagging plays vital role in feature extraction process.
Example:

Input: 'such', an', awesome', idea', the', continual', learning', program',' with', 'kindle', 'I', 'am',' happy', 'hmm', 'sleep'

Output: ('such', 'JJ'), ('an', 'DT'), ('awesome', 'JJ'), ('idea', 'NN'), ('the', 'DT'),('continual', 'JJ'), ('learning', 'NN'), ('program', 'NN'), ('with', 'IN'), ('kindle', 'JJ'), ("I", 'PRP'), ('am', 'VBP') ,('happy', 'JJ'), ('hmm', 'NN'), ('sleep', 'NN')

\subsection{Knowledge Base Preparation}

Here the knowledge base preparation is in terms of features. Features are very important in any text classification problem. Converting source documents into a representation that the task that is being addressed.

\subsubsection{Knowledge Extraction:}

Knowledge/Feature extraction is by using POS tagging. All tags are not the emotional words, to know the emotion carrying words we have referred the Russell's Circumplex Model of Affect [15].

Circumplex Model: The circumplex model suggests that emotions are distributed in a two-dimensional circular space, containing pleasure (valence) and activation (arousal) dimensions. The activation dimension measures if one is likely to take an action in a mood state. The pleasure dimension measures how positive or negative a person feels. The vertical axis represents activation or arousal, and horizontal axis represents pleasure or valence. The center of the circle represents a neutral valence and a medium level of arousal. It consists of 28 affect words [15] shown in figure 3 , considered these as core affect words.

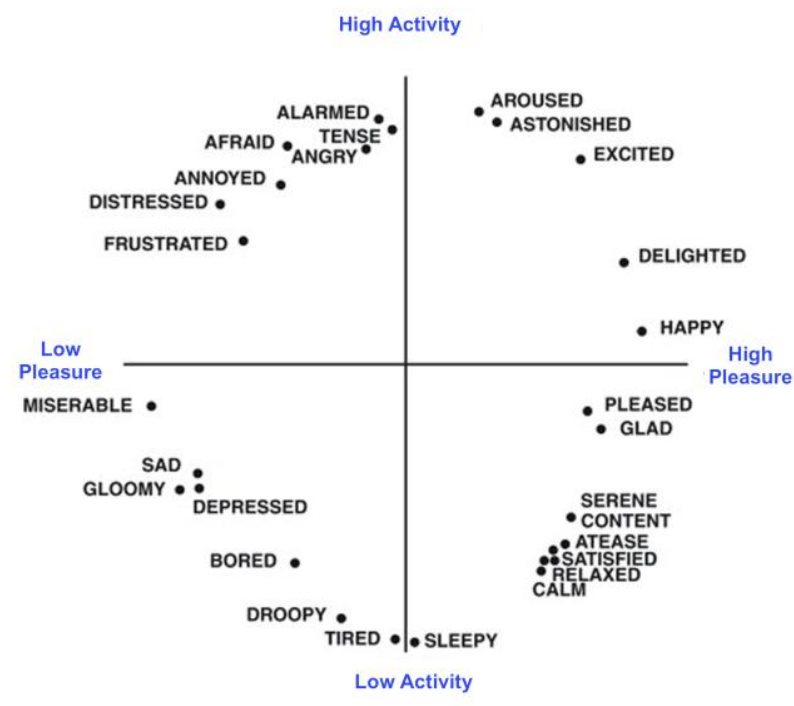

Fig .3 Circumplex Model of Affect by J. A. Russell

Extended Words: Observed that the emotion carrying words are mainly nouns, adjectives, verbs, adverbs. Later extended the features/knowledge according to the dataset, here we have considered adjective phrases, noun phrases, few adverbs, and verbs along with core affect words.

$\begin{array}{lll}\text { JJ - adjective } & & \text { JJ-NN } \\ \text { NN - noun } & & \text { NN-NN } \\ \text { RB - adverb } & \text { JJ-JJ } \\ \text { VB - verb } & \text { RB-JJ } & \end{array}$


Negation: The words with preceded by negated word can be called as negation features for example "not happy", "cannot sleep". The negation features have the ability to completely show the opposite side of a tweet. The negation features changes the semantic of the sentence, so these features are important.

\subsubsection{Feature Selection}

All the specified tags may not be useful. If every specified tag is considered the feature space increases dramatically, this results in curse of dimensionality as a result the number of tweets required increases exponentially.

\section{Curse of Dimensionality Reduction}

Consider more frequently occurred words in the dataset as features. As a result, feature space does no longer include all the words, but instead it only contains the frequently occurred emotion carrying words. This method reduces the size of feature space dramatically, without losing informative terms.

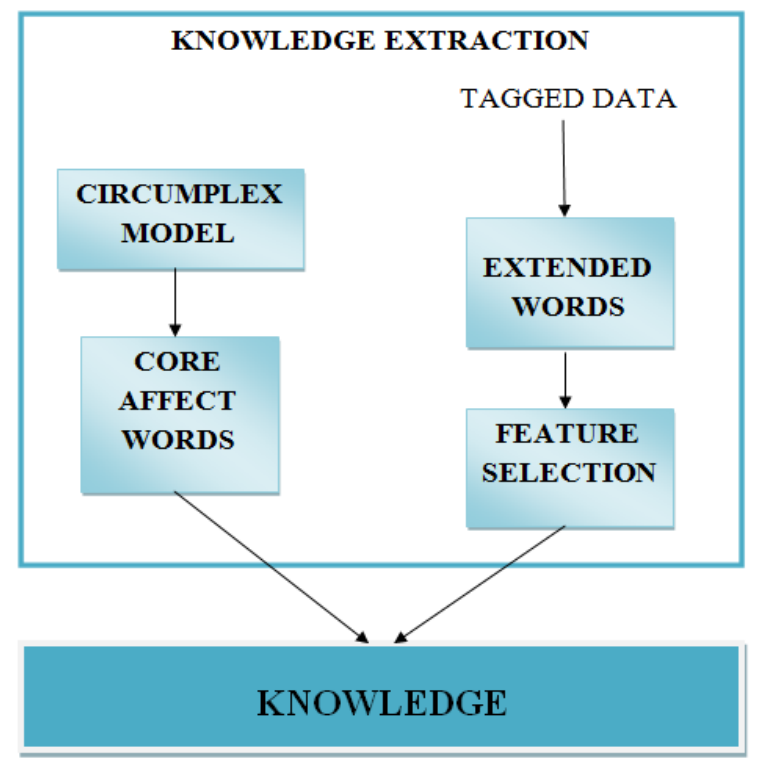

Fig. 4 Workflow of Knowledge Base Preparation

\subsection{Knowledge Base Validation}

Knowledge base validation is by using defined dictionary which is created by using standard weighted lexicon version 3.0 given by Princeton University [16].

The lexicon consists of 6 fields, they are:

- First field is word category - the categories considered in this dictionary are adjective, adverb, noun, verb

- $\quad$ Second field is POSID - unique number

- $\quad$ Third field is +ve Score - Positive Score given to word

- $\quad$ Fourth field is -ve Score - Negative Score given to word

- Fifth field is SynsetTerms - Similar words with sense number

- $\quad$ Sixth field is Gloss - Glossary

Existing lexicon is converted to four column dictionary using pattern matching techniques. The dictionary's 4 columns are:
1. First column:- Word

2. Second column :- Category of word

3. Third column:- Positive Score of word

4. Fourth column:- Negative Score of word

The features/knowledge base is checked against the dictionary for its score.

Conditions: - The condition says that the feature with positive score may come under Happy-Active Class or Happy-Inactive Class and the feature with negative score may come under Unhappy-Active Class or Unhappy-Inactive Class.

Condition 1:- The word with high positive score (0.5-1.0) will come under Happy-Active Class.

Condition 2:- The word with less positive score (0.1-0.5) will come under Happy-Inactive Class.

Condition 3:- The word with less negative score(- 0.1 to -0.5$)$ will come under Unhappy-Active Class.

Condition 4:- The word with high negative score (- 0.5 to 1.0) will come under Unhappy-Inactive Class.

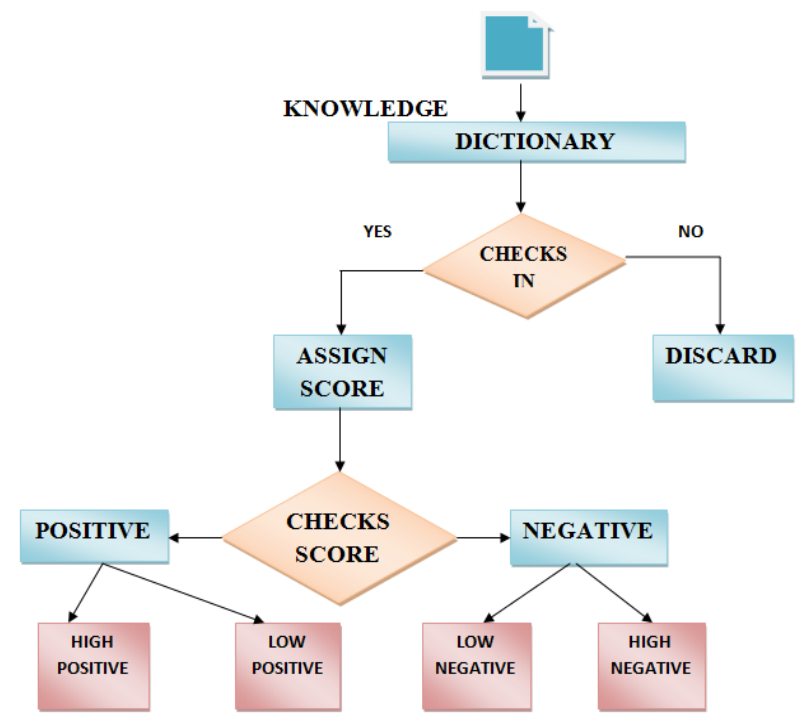

Fig.5 Workflow of Knowledge Validation

Seedlist: - The extracted and validated features are stored on to a list called seedlist along with the category.

\subsection{Classification}

Rule based approach is used to classify the tweets under specified class categories with the help of knowledge base. The knowledge base applies certain rules to get a conclusion. The emotional words in the tweet will fall under some category, if more number of words in a tweet falls under any of the specified category then that class label is assigned for the tweet. The system consists of four different classes. They are:

C1 - Happy-Active Class

C2 - Happy-Inactive Class

C3 - Unhappy-Active Class

C4 - Unhappy-Inactive Class 
- The happy-active class specifies more happy emotions like :'sohappy', 'excited', enjoy', 'excited', 'proud', 'amazing', 'delighted', 'wonderful etc.

- The happy-inactive class specifies less happy emotions like: 'calm','peace','quiet','silent','convinced','content','satis fied','relaxing','resting' etc.

- The unhappy-active class specifies less sad emotions like: 'nervous','anxious','tennsion','afraid','fear','angry','fur ious','bother','disturbed','mad' etc.

- The unhappy-inactive specifies more sad emotions like: 'sad', 'miserable', 'verysad', 'sorrow', 'disappointed' etc.

\section{EXPERIMENTS and RESULTS}

The system consists of four classes of emotions namely (c1) Happy-Active, (c2) Happy-Inactive, (c3) Unhappy-Active, and (c4) Unhappy-Inactive.

Table 1. Number of tweets with accuracy

\begin{tabular}{|c|c|}
\hline Approach & Rule Based \\
\hline No. of tweets & 1048576 \\
\hline Overall Accuracy & $85.1 \%$ \\
\hline
\end{tabular}

The system classifies the complete 1 million records, it gives the accuracy over $85.1 \%$.

\subsection{Cross-validation}

In the rule based system, classified the 1 million records, it gives the accuracy over $85 \%$. Validated using nonexhaustive cross-validation called $\mathrm{k}$-fold cross validation with 10 -folds which are randomly taken.

Table 2. Random Samples with Accuracy

\begin{tabular}{|c|c|}
\hline SAMPLES & ACCURACY \\
\hline Fold 1 & $80 \%$ \\
\hline Fold 2 & $92.1 \%$ \\
\hline Fold 3 & $84 \%$ \\
\hline Fold 4 & $80 \%$ \\
\hline Fold 5 & $80 \%$ \\
\hline Fold 6 & $90 \%$ \\
\hline Fold 7 & $80.4 \%$ \\
\hline Fold 8 & $85 \%$ \\
\hline Fold 9 & $94.2 \%$ \\
\hline Fold 10 & $86.2 \%$ \\
\hline Total & 85.1 \\
\hline
\end{tabular}

\subsection{Evaluation Metrics}

The execution of the emotion detection system is appraised by implementing the system on test data. The Performance of emotion detection system is then evaluated based on Precision, Recall and F-measure score obtained.

4.2.1 Precision: Precision is also referred to as positive predictive value.

$$
\text { Precision }=\mathrm{TP} /(\mathrm{TP}+\mathrm{FP})
$$

Where, TP and FP are the number of true positive and false positive predictions for the considered class

4.2.2 Recall: Recall is commonly referred to as sensitivity, corresponds to the true positive rate of the considered class.

$$
\text { Recall }=\mathrm{TP} /(\mathrm{TP}+\mathrm{FN})
$$

Table 3. Precision and Recall

\begin{tabular}{|c|c|c|}
\hline Category & Precision & Recall \\
\hline C1 & 0.66 & 0.80 \\
\hline C2 & 0.79 & 0.92 \\
\hline C3 & 0.90 & 0.80 \\
\hline C4 & 0.94 & 0.90 \\
\hline
\end{tabular}

The above table gives the precision and recall of each class.

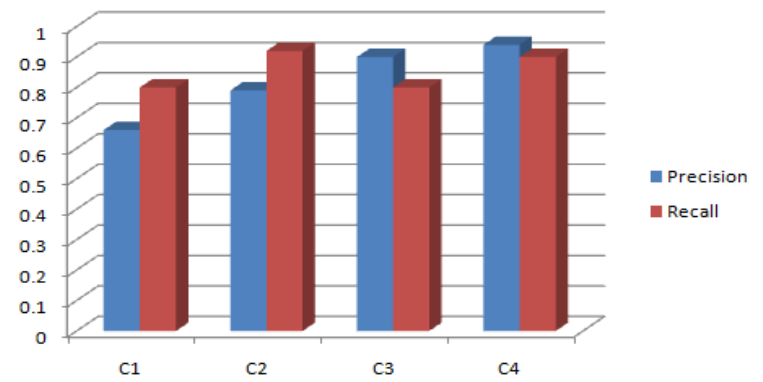

Fig.6 Precision and Recall

\subsubsection{F-Measure:}

The F-score is a measure of test's accuracy.

$$
F_{1}=2 . \frac{\text { precision } \text {. recall }}{\text { precision }+ \text { recall }}
$$

Table 4. F-measure

\begin{tabular}{|c|c|}
\hline Category & F-measure \\
\hline $\mathrm{C} 1$ & 0.72 \\
\hline $\mathrm{C} 2$ & 0.85 \\
\hline $\mathrm{C} 3$ & 0.85 \\
\hline $\mathrm{C} 4$ & 0.92 \\
\hline
\end{tabular}

The above table gives the F-measure. 


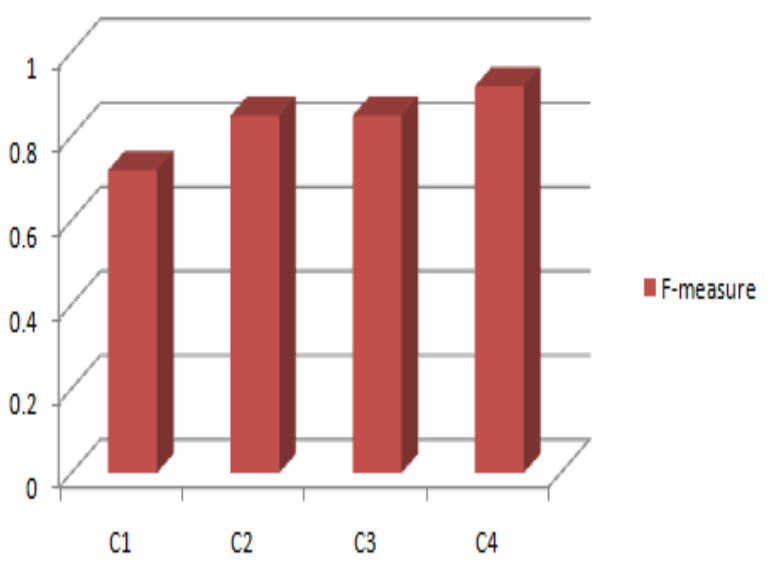

Fig. 7 F-measure

\section{CONCLUSION AND FUTURE WORK}

The proposed approach enables us to classify large amounts of short texts messages into four classes of emotion. Classifying short texts according to finer-grained classes of emotions provides rich and informative data about the emotional states of individuals. This system can be used by health care professionals for early detection of the psychological disorders such as anxiety or depression. This system can also be useful in educational institutions and also in intelligence bureau. In this system we have considered only the text part and emoticons are ignored, emoticons also play major role in the detection of emotion, in near future hope to include emoticons along with text The system focused only on English sentences, but Twitter has many international users. It should be possible to use this system to classify emotions in other languages.

\section{REFERENCES}

[1] Maryam Hasan, Elke Rundensteiner, and Emmanuel Agu, May 2014, "EMOTEX: Detecting Emotions in Twitter Messages," ASE BIGDATA/SOCIALCOM/CYBERSECURITY Conference, 27-31.

[2] Johan Bollen, Huina Mao, and Alberto Pepe, 2011, "Modeling public mood and emotion: Twitter sentiment and socio-economic phenomena," in International AAAI Conference on Weblogs and Social Media (ICWSM'11).

[3] Mike Thelwall, Kevan Buckley, and Georgios Paltoglou, 2007, "Sentiment in twitter events," Journal of the American Society Tavel, Modeling and Simulation Design. AK Peters Ltd, P.

[4] Ed Diener and Martin E. P. Seligman, 2004, "Beyond money: toward an economy of well-being," in
PSYCHOLOGICAL SCIENCE IN THE PUBLIC INTEREST, American Psychological Society.

[5] Ed Diener, 2009, Assessing well-being: The collected works of Ed Diener, vol. 3, Springer,

[6] Shigehiro Oishi Ed Diener Ed Diener, "Subjective wellbeing: The science of happiness and life satisfaction,"

[7] Minsu Park, Chiyoung Cha, and Meeyoung Cha, 2012, "Depressive moods of users portrayed in twitter," in Proc. of the ACM SIGKDD Workshop on Healthcare Informatics, HI-KDD

[8] Munmun De Choudhury, Michael Gamon, Scott Counts, and Eric Horvitz , 2013, "Predicting depression via social media.," in International AAAI Conference on Weblogs and Social Media (ICWSM'13), The AAAI Press.

[9] Golder S, Loke YK, Bland M, 2011, Meta-analyses of Adverse Effects Data Derived from Randomized Controlled Trials as Compared to Observational Studies: Methodological Overview. PLoS Med 8(5): e1001026. doi:10.1371/journal.pmed.1001026.

[10] Munmun De Choudhury, Scott Counts, and Michael Gamon, 2012, "Not all moods are created equal! Exploring human emotional states in social media," in Sixth International AAAI Conference on Weblogs and Social Media (ICWSM'12).

[11] Carlo Strapparava and Rada Mihalcea, 2008, "Learning to identify emotions in text," in Proceedings of the 2008 ACM symposium on Applied computing. ACM, pp.1556-1560.

[12] M. Naaman, J. Boase, and C.-H. Lai. 2010, Is it Really About Me? Message Content in Social Awareness Streams. In ACM Conference on Computer Supported Cooperative Work (CSCW)

[13] D. Kleinbaum, L. Kupper, and K. Muller. 2007, Applied regression analysis and other multivariable methods. Duxbury Pr.

[14] Go, A., Bhayani, R., \& Huang L. 2009, Twitter Sentiment Classification Using Distant Supervision. Retrieved December 6, 2014, from http://cs.stanford.edu/people/alecmgo/papers/TwitterDist antSupervision09.pdf

[15] J. A. Russell, 1980, "A circumplex model of affect, 1980," Journal of Personality and Social Psychology, vol. 39, pp. 1161-1178.

[16] http://sentiwordnet.isti.cnr.it 